\title{
Genome-wide association study of rust traits in orchardgrass using SLAF-seq technology
}

\author{
Bing Zeng ${ }^{1{ }^{*}}$, Haidong Yan ${ }^{2 \dagger}$, Xinchun Liu ${ }^{3}$, Wenjing Zang ${ }^{4}$, Ailing Zhang ${ }^{2}$, Sifan Zhou ${ }^{2}$, Linkai Huang ${ }^{2^{*}}$ \\ and Jinping Liu ${ }^{4}$
}

\begin{abstract}
Background: While orchardgrass (Dactylis glomerata L.) is a well-known perennial forage species, rust diseases cause serious reductions in the yield and quality of orchardgrass; however, genetic mechanisms of rust resistance are not well understood in orchardgrass.

Results: In this study, a genome-wide association study (GWAS) was performed using specific-locus amplified fragment sequencing (SLAF-seq) technology in orchardgrass. A total of 2,334,889 SLAF tags were generated to produce 2,309,777 SNPs. ADMIXTURE analysis revealed unstructured subpopulations for 33 accessions, indicating that this orchardgrass population could be used for association analysis. Linkage disequilibrium (LD) analysis revealed an average $r^{2}$ of 0.4 across all SNP pairs, indicating a high extent of LD in these samples. Through GWAS, a total of 4,604 SNPs were found to be significantly $(P<0.01)$ associated with the rust trait. The bulk analysis discovered a number of 5,211 SNPs related to rust trait. Two candidate genes, including cytochrome P450, and prolamin were implicated in disease resistance through prediction of functional genes surrounding each high-quality SNP $(P<0.01)$ associated with rust traits based on GWAS analysis and bulk analysis
\end{abstract}

Conclusions: The large number of SNPs associated with rust traits and these two candidate genes may provide the basis for further research on rust resistance mechanisms and marker-assisted selection (MAS) for rust-resistant lineages.

Keywords: Genome-wide association study, Orchardgrass, Rust disease, SLAF-seq

\section{Background}

Orchardgrass (Dactylis glomerata L.) is a cool-season forage grass that is wildly cultivated in all mild, subtropical regions of the world [1]. Due to its high yield, high nutritional value, and shade tolerance, it has become a valuable pasture species in China [2]. However, orchardgrass is frequently infected by rust disease, which leads to low yield and poor quality [3].

Several studies on rust disease in orchardgrass have been reported. Tajimi et al. [4] studied orchardgrass clonal lines and found that the rusts of orchardgrass,

\footnotetext{
* Correspondence: zbin78@163.com; huanglinkai@sicau.edu.cn

${ }^{\dagger}$ Equal contributors

'Department of Animal Science, Southwest University, Rongchang, Chongqing 402460, China

2Department of Grassland Science, Animal Science and Technology College,

Sichuan Agricultural University, Chengdu 611130, China

Full list of author information is available at the end of the article
}

timothy, and ryegrass were closely related and that stem rust was under the genetic control of orchardgrass. Miller and Carlson [5] evaluated rust resistance in orchardgrass based on both phenotypic performance and a polycross progeny test through phenotypic selection. Yan et al. [6] investigated 242 germplasm resources of orchardgrass for 2 years, finding that 13 accessions exhibited high resistance to rust disease. However, the phenotype of an organism can be influenced by both its genotype and its environment. To better assess the impact of this disease, additional methods are needed to study the desirable genetic traits for these plants.

Genome-wide association studies (GWASs) have become one of the most commonly strategies for identifying genes underlying complex traits in plants. In model species, such as Arabidopsis thaliana, the potential of GWAS to reveal genetic traits has been demonstrated 
successfully by the functional validation of the accelerated cell death 6 (ACD6) gene [7]. This approach has also been used to develop a precise estimation of variation in traits such as flowering time [8] of Arabidopsis thaliana, leaf architecture [9] of maize (Zea mays), and blight resistance [10] of wheat (Triticum aestivum L.).In recent studies on rice (Oryza sativa), several genes that have large effects on traits involved in determining yield, morphology, and salinity tolerance have been identified [11-13]. In the past few years, flowering time genes in barley (Hordeum vulgare) [14], the PsyI-AI locus in wheat [15], and the rhg-1 gene in soybean (Glycine max) [16] have been identified as candidate genes [17] through GWAS. Rust disease has seriously influenced plant growth and development, so some studies focus on conducting GWAS to plant rust disease. For example, a GWAS was conducted using 232 winter wheat breeding lines to identify loci conferring resistance to Ug99 that is a new race of stem rust and threatens global wheat production, and 12 loci associated with Ug99 resistance were discovered in this study [18]. A number of 177 oat (Avena sativa) accessions were evaluated for disease resistance and further genotyped with 15,000 Diversity Arrays Technology (DArT) and 31 simple sequence repeat and markers to disclose association with disease resistance trait and found five markers were associated with rust resistance [19].

Consequently, several reduced-representation sequencing technologies, including restriction site-associated sequencing (RADseq), double digest RADseq, and twoenzyme genotyping-by-sequencing (GBS), were developed as cost-effective methods for SNP discovery and high-throughput genotyping [20]. Recently, specificlocus amplified fragment sequencing (SLAF-seq), a more efficient solution for large-scale genotyping, was developed by Sun et al. [21]. This approach involves several distinguishing characteristics: (1) fine-mapping resolution among founders can be performed given a highdensity of genotypes; (2) outcrossing reshuffles variation in the founder genomes, providing some control over population structure effects; (3) joint-linkage mapping identifies low-resolution quantitative trait loci (QTLs) across all recombinant inbred line (RIL) families, and this genetic background can be controlled while performing nested associations for fine mapping; and (4) the use of RILs allows repeated measurement of phenotypes on the same lines in common and different environments [22]. This approach has previously been used successfully to perform GWAS [23].

SLAF-seq is highly automated due to the development of bioinformatics tools and high-throughput sequencing technology applications. SLAF sequencing methods ascertain the uniformity, efficiency, and density of marker development, and they have been applied in several studies. For example, Li et al. [24] constructed a high-density soybean genetic map to discover QTLs pertaining to isoflavone content that were consistent across various environments; Wei et al. [25] established a high-density SNP map for cucumber (Cucumis sativus L.) through SLAF-seq to detect fruitrelated QTLs. Additional studies have created high-density genetic maps in order to provide a platform for gene/QTL fine-mapping $[20,26,27]$. These studies showed that SLAF-seq methods are used primarily to construct genetic maps for discovering valuable QTLs; however, for QTL mapping, a large investment of time is needed to establish mapping populations, and the intensive labor required may be a limitation for identification of specific traits. To avoid the limitations of this approach, GWAS is a reliable method used to confirm molecular markers relating to important economic traits. SNP markers that cover the entire genome may be analyzed without the construction of mapping populations [28]. Moreover, several studies have used used SLAF-seq for GWAS [23, 29-31].

In this study, we use the SLAF-seq technology to perform a GWAS of rust traits in orchardgrass to identify the associated SNPs and predict functional genes. These results will provide a basis for orchardgrass breeding and may be helpful in enhancing rust resistance in orchardgrass.

\section{Results and Discussion}

\section{Sequence and quality statistics}

A total of $76.74 \mathrm{M}$ reads were obtained from this experiment. The average Q3 value was $86.91 \%$, and the average GC content was $46.97 \%$. Details are listed in Additional file 1: Table S1.

\section{Specific-locus amplified fragment sequencing results}

In total, $76.74 \mathrm{M}$ reads were generated for the 33 genotypes, encompassing $4.30 \mathrm{~Gb}$ of the orchardgrass genomic DNA sequence. A total of 2,334,889 SLAF tags were identified throughout the genome (Table 1). These SLAF tags were divided into three types: Marker (polymorphism tags), No ploy (no polymorphism tags), and Repeat (SLAF tags on repeat sequences) (Table 1). PLINK (v1.07) [32] was used to carry out quality control of the data. Those SNPs with low integrity $(<85 \%)$, low minor allele frequency $(<5 \%)$, and insufficient reads $(<90 \%)$ were discarded. After filtration, 2,309,777 SNPs were used for further analysis.

Table 1 The statistical results for each type of SLAF tags

\begin{tabular}{lllll}
\hline Type & Marker & NoPoly & Repeat & Total \\
\hline Number & 643008 & 1689409 & 2472 & 2334889 \\
Percent & $27.54 \%$ & $72.36 \%$ & $0.11 \%$ & $100 \%$ \\
\hline
\end{tabular}

Note: Marker, pleomorphic tags; Nopoly, non-pleomorphic tags; Repeat: SLAF tags on repeat sequence 


\section{Phylogenetic analysis}

The 33 orchardgrass samples could not be clearly divided into subgroups by the neighbor-joining analysis using MEGA software. However, the results from this phylogenetic analysis showed that the HS (high susceptibility of disease) materials were relatively concentrated, comparing with HR (high level of resistance of disease) materials (Fig. 1).

\section{Group structure and clustering analysis}

The best dataset was produced by using a K-value of 2, indicating that our samples probably derived from 2 ancestors. With the maximum membership probability, 5 accessions were assigned to group 1 (G1), while 28 accessions were assigned to group 2 (G2). All 5 accessions from G1 were HR samples, and all 16 HS accessions (16) were included in G2 (Fig. 2).

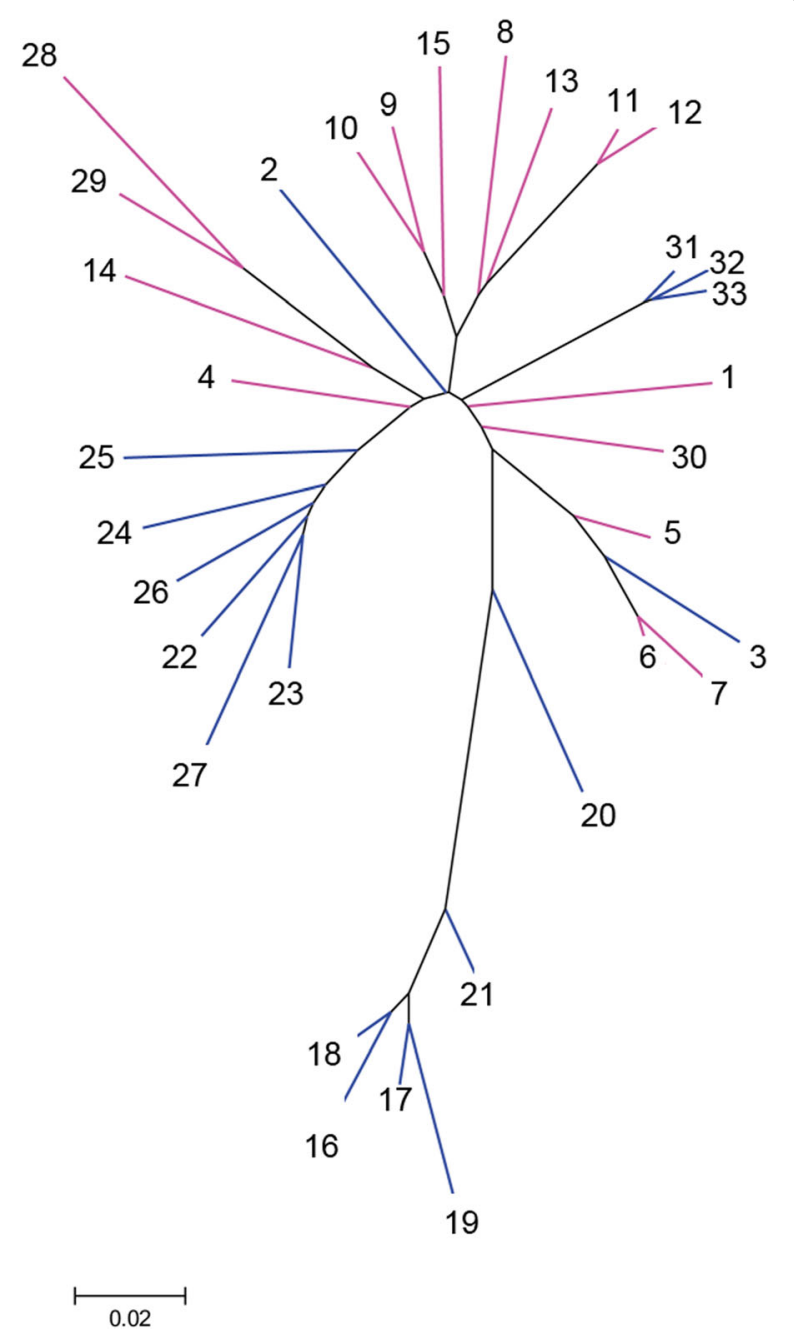

Fig. 1 The evolutionary tree of 33 orchardgrass accessions. The branch indicates each material. The red line is HS materials, and blue line is HR materials
We applied a clustering strategy to the samples with ADMIXTURE software. This method has been used with large sample sizes, exhibiting a strong capability to assign individuals into populations. The best dataset $(K=2)$ showed that our samples probably derive from two ancestors. It is important to use population-based methods to separate accessions from mixed populations into unstructured subpopulations, allowing for association analyses between phenotypes and molecular bands to be conducted in homogeneous subpopulations [33, 34]. In this study, the accessions associated with resistant and susceptible traits remained in G1, indicating that this orchardgrass population could be used for association analysis.

\section{PCA analysis}

A principal component analysis (PCA) was performed using the 2,309,777 SNPs from all 33 accessions to estimate the clusters of population. Principal Component 1 (PC1) explained $7.94 \%$ of the variation in the genotypic data, while PC2 and PC3 explained $5.20 \%$ and $2.57 \%$ of the variation, respectively. Although there are intermediate accessions that make the groupings less clear, the PCA results indicate that the HS samples can be clustered into one group (Fig. 3).

Based on analysis of 2,309,777 SNPs, a neighborjoining tree was constructed using MEGA software. The results from the phylogenetic analysis showed that the 33 orchardgrass samples could not be clearly divided into two major clusters (Fig. 1), though the 16 HS individuals were able to cluster into one group as shown in the PCA results (Fig. 3). This indicates that there may be no strong relationship between rust resistance and genetic background. Several individuals appeared to be intermediates between the two groups, making the distinction between groups less clear; it may be that we lack a sufficient number of individuals in our sample to appropriately delineate the groups.

\section{Linkage disequilibrium analysis}

The 2,309,777 SNPs generated from the 2,334,889 SLAF markers with unknown chromosome information were used to evaluate the extent of LD in the 33 orchardgrass accessions. In total, 9,345,646 pairs of SNPs were detected and an $\mathrm{r}^{2}$ value was calculated for each pair. The values ranged from 0.0000 to 1.0000 , with an average $\mathrm{r}^{2}$ of 0.4, indicating a substantial amount of LD existing in these SNPs. Of the 9,345,646 pairs, 7,270 $(0.08 \%)$ were considered to be in LD with strong linkage at $r^{2}>0.8$ (Additional file 2).

\section{GWAS analysis and bulk analysis}

The association analysis between SNP markers and the rust trait was performed using TASSEL to search for associated tags and allelic variation. After general 


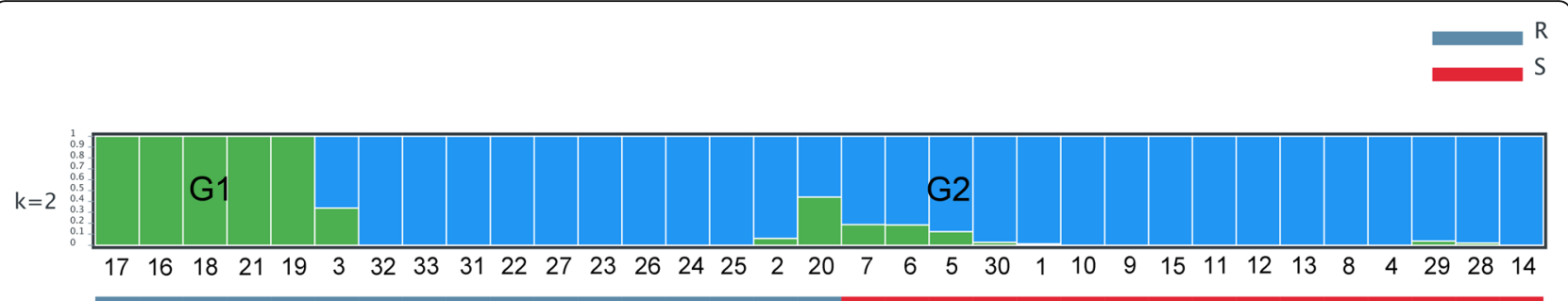

Fig. 2 Two subgroups inferred from ADMIXTURE analysis. The vertical coordinated of each subgroup means the membership coefficients for each accession. The digits of the horizontal coordinate represent the accessions according to Table 3. Green zone: G1; Blue zone: G2. The blue line means HR materials; The red line means HS materials

linear model (GLM) analysis of 2,309,777 SNPs, 4,604 SNPs were significantly $(P<0.01)$ associated with the rust trait (Additional file 3). At a more stringent Pvalue cutoff of $<0.001,1,761$ SNPs were still associated with the rust trait (Additional file 4). The bulk analysis was conducted to discover 69,690 SNPs related to the rust trait, and a total number of 5,211 SNPs were filtered when the SNP index is over 0.3 (Additional file 5).

\section{Bioinformatics analysis of candidate genes}

We predicted 555 genes from these 4,604 SNPs in our study (Additional file 6) through GWAS analysis, and only four candidate genes, including cytochrome P450, Pc68LrkC4, prolamin, and EF-hand $\mathrm{Ca}^{2+}$-binding protein (TaCab1), were implicated with disease resistance in the Nonredundant NCBI nucleotide sequences (NT) database, and their predicted molecular functions and biological processes are specified in Table 2.

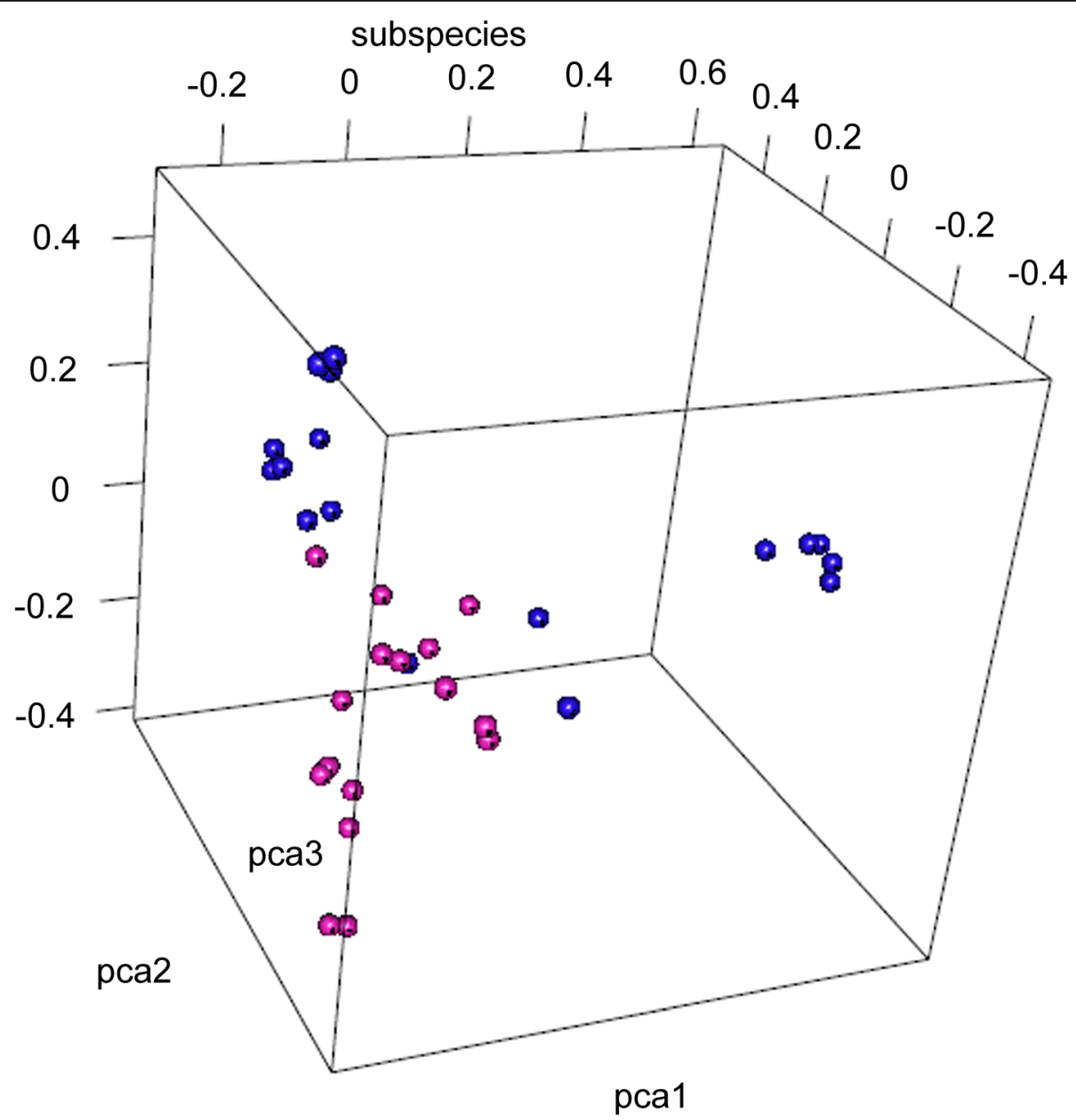

Fig. 3 The PCA analysis of 33 orchardgrass accessions. The red balls mean HS materials; the blue balls mean HR materials 
Table 2 Bioinformatics analysis of candidate genes

\begin{tabular}{llll}
\hline SLAF marker & E_value & Nearest gene & Predict Function \\
\hline Marker10747 & $5.00 \mathrm{E}-18$ & Cytochrome P450 & Resist pathogenic bacterial \\
Marker11029 & $2.00 \mathrm{E}-12$ & Cytochrome P450 & Resist pathogenic bacterial \\
Marker13729a & $2.00 \mathrm{E}-17$ & Cytochrome P450 & Resist pathogenic bacterial \\
Marker1700 & $5.00 \mathrm{E}-13$ & Cytochrome P450 & Resist pathogenic bacterial \\
Marker3124 & $5.00 \mathrm{E}-18$ & Cytochrome P450 & Resist pathogenic bacterial \\
Marker33680 & $5.00 \mathrm{E}-18$ & Cytochrome P450 & Resist pathogenic bacterial \\
Marker40081 & $2.00 \mathrm{E}-11$ & Cytochrome P450 & Resist pathogenic bacterial \\
Marker4015 & $2.00 \mathrm{E}-27$ & Cytochrome P450 & Resist pathogenic bacterial \\
Marker4728 & $2.00 \mathrm{E}-27$ & Cytochrome P450 & Resist pathogenic bacterial \\
Marker5389 & $1.00 \mathrm{E}-08$ & Cytochrome P450 & Resist pathogenic bacterial \\
Marker12565 & $6.00 \mathrm{E}-22$ & Pc68LrkC4 & Activate chemical reactions \\
Marker2312943 & $8.00 \mathrm{E}-16$ & Pc68LrkC4 & Activate chemical reactions \\
Marker2649 & $1.00 \mathrm{E}-24$ & Prolamin gene & Regulate stripe rust resistance \\
Marker10408 & $1.00 \mathrm{E}-08$ & Prolamin gene & Regulate stripe rust resistance \\
Marker21044 & $8.00 \mathrm{E}-21$ & TaCab1 & Regulate stripe rust resistance \\
\hline
\end{tabular}

Note: ${ }^{a}$ means that makers have also been found in bulk analysis

Four genes, including cytochrome P450, Pc68LrkC4, prolamin, and $\mathrm{TaCab} 1$, were discovered by detecting genes surrounding each candidate SNP $(<1 \mathrm{Mb})$. Cytochrome $\mathrm{P} 450$ plays an important role in the synthesis of secondary metabolites of fungi and metabolism of foreign compounds [35]. Studies on Populus tomentosa infected by stripe rust showed that Cytochrome P450 is one of the genes related to germ defense [36]. Pc68LrkC4 was isolated from Avena sativa and contains a retrotransposon and repetitive DNA linked to a receptor kinase gene. It has been shown that the leaf rust resistance gene Lr10 of Triticum aestivum encodes an extracellular receptor protein kinase with similar domains [37]. Several studies on prolamin have found that proteins with the same or similar rust resistance are closely related to prolamin, and most of these cluster in dendrograms. For example, wheat stripe rust resistance was related to the genetic distance of prolamin, guessing it might because wheat stripe rust gene and partial encoded gene of prolamin were chained on 1BS [38, 39]. In affinity reactions between wheat and stripe rust, the expression of the TaCab1 gene increases significantly. At addition, its expression can be induced by several different biotic stresses. The TaCab1 gene exhibits a marked change in expression after being treated with $\mathrm{CaCl} 2$. Researchers have guessed that TaCab1 plays a role in the interaction between wheat and stripe rust via $\mathrm{Ca}^{2+}$ transport. In addition, TaCab1 gene silencing increases wheat's resistance to stripe rust. Therefore, researchers have shown that the TaCab1 gene is correlated with susceptibility to stripe rust [40]. In conclusion, these four candidate genes play important roles in disease progression, particularly the
TaCab1 gene. This indicates that these candidate genes are likely to be critical in orchardgrass resistance to rust disease.

We also associated the results between GWAS analysis and bulk analysis to confirm these four predicted genes; however, two types of analysis only shared three markers representing two predicted genes including Cytochrome P450 and Prolamin gene (Table 2). Therefore, these two genes might be more reliable for regulation mechanism of rust resistance in orchgardgrass.

\section{Method}

\section{Experimental materials}

A total of 33 orchardgrass accessions, with high level of resistance of disease (HR) and high susceptibility of disease (HS), as evaluated by Yan et al. [6], were chosen for the experiment, including 17 with HR and 16 with HS in 2011 or 2012 (Table 3). The germplasm of orchardgrass used in this study consisted of 33 accessions that originated from Asia, Africa, Europe, and Oceania. For each accession, 10 individuals were randomly, and $0.5 \mathrm{~g}$ of clean young leaves were selected per plant in 2014 for further DNA extraction.

\section{Specific-locus amplified fragment sequencing}

Total orchardgrass genomic DNA was extracted using the DNeasy Plant Mini Kit (Qiagen USA); the quality and quantity of DNA was then inspected using $0.8 \%$ gel electophoresis. The quantified DNA was diluted to $20 \mu \mathrm{g} / \mu \mathrm{L}$ and was stored at $-20{ }^{\circ} \mathrm{C}$ before use.

The orchardgrass genomic DNA was analyzed with SLAF-seq [21]. Sequencing libraries of each accession 
Table 3 The detailed information about accession number, name, origin, and rust resistance in orchardgrass for 2011 and 2012

\begin{tabular}{|c|c|c|c|c|}
\hline Number & Accession name & Origin & 2011 & 2012 \\
\hline 1 & 01819-6 & Beijing,China & $\mathrm{HS}$ & $\mathrm{HS}$ \\
\hline 2 & $01824-2$ & Beijing,China & $H R$ & $H R$ \\
\hline 3 & $02122-5$ & Hubei,China & $H R$ & $H R$ \\
\hline 4 & 231469-1 & Libya & $\mathrm{HS}$ & HS \\
\hline 5 & $2410-2$ & Xinjiang,China & $\mathrm{HS}$ & HS \\
\hline 6 & $2410-6$ & Xinjiang,China & $\mathrm{HS}$ & HS \\
\hline 7 & $2410-7$ & Xinjiang,China & $\mathrm{HS}$ & HS \\
\hline 8 & 287804-1 & Spain & $\mathrm{HS}$ & HS \\
\hline 9 & $287804-2$ & Spain & $\mathrm{HS}$ & HS \\
\hline 10 & 287804-3 & Spain & $\mathrm{HS}$ & HS \\
\hline 11 & $287804-4$ & Spain & $\mathrm{HS}$ & HS \\
\hline 12 & $287804-5$ & Spain & $\mathrm{HS}$ & HS \\
\hline 13 & $287804-8$ & Spain & $\mathrm{HS}$ & HS \\
\hline 14 & 292587-1 & Israel & $\mathrm{HS}$ & HS \\
\hline 15 & $302884-3$ & Spain & $\mathrm{HS}$ & HS \\
\hline 16 & 308794-1 & India & $\mathrm{HR}$ & $\mathrm{HR}$ \\
\hline 17 & $308794-2$ & India & $\mathrm{HR}$ & $\mathrm{HR}$ \\
\hline 18 & $308794-3$ & India & $\mathrm{HR}$ & $\mathrm{HR}$ \\
\hline 19 & $308794-5$ & India & $\mathrm{HR}$ & $H R$ \\
\hline 20 & $308794-7$ & India & $\mathrm{HR}$ & $H R$ \\
\hline 21 & $308794-8$ & India & $\mathrm{HR}$ & $H R$ \\
\hline 22 & $325293-2$ & Russian Federation & $\mathrm{HR}$ & $\mathrm{HR}$ \\
\hline 23 & $325293-4$ & Russian Federation & $\mathrm{HR}$ & $H R$ \\
\hline 24 & $325293-5$ & Russian Federation & $H R$ & $H R$ \\
\hline 25 & $325293-6$ & Russian Federation & $\mathrm{HR}$ & $\mathrm{HR}$ \\
\hline 26 & $325293-7$ & Russian Federation & $H R$ & HR \\
\hline 27 & $325293-8$ & Russian Federation & $\mathrm{HR}$ & $\mathrm{HR}$ \\
\hline 28 & $578635-7$ & Morocco & $\mathrm{HS}$ & HS \\
\hline 29 & $578635-8$ & Morocco & $\mathrm{HS}$ & $\mathrm{HS}$ \\
\hline 30 & $79-118-2$ & Netherlands & $\mathrm{HS}$ & HS \\
\hline 31 & woronowii(H12)-3 & New Zealand & $\mathrm{HR}$ & $\mathrm{HR}$ \\
\hline 32 & woronowii(H12)-4 & New Zealand & $\mathrm{HR}$ & $\mathrm{HR}$ \\
\hline 33 & woronowii(H12)-7 & New Zealand & $H R$ & HR \\
\hline
\end{tabular}

Note: $H R$ high resistance, $H S$ high sense of disease

were constructed through digestion with the restriction enzyme HaeIII, (New England Biolabs, USA). A single nucleotide (A) overhang was added to the digested fragments with Klenow Fragment $\left(3^{\prime} \rightarrow 5^{\prime}\right.$ exo-) (New England Biolabs, USA) and dATP at $37{ }^{\circ} \mathrm{C}$, and then duplex taglabeled sequencing adapters (PAGE purified, Life Technologies, Beijing, China) were ligated to the A-tailed DNA with T4 DNA ligase. The PCR products were purified using Agencourt AMPure XP beads (Beckman Coulter, High Wycombe, UK) and pooled. The pooled sample was separated via electrophoresis in a 2\% agarose gel. Fragments with indexes and adaptors from 400 to 450 bp were excised and purified using a QIAquick Gel Extraction Kit (QIAGEN, Duesseldorf, Germany). Finally, the gelpurified product was sequenced using the Illumina HiSeq 2500 system (Illumina, Inc., San Diego, CA, USA). The average depth of sequencing was 6.7 $\times$. After sequencing, low-quality reads were filtered out (quality score $<20$ ). Reads with double ends were compared with similar sequences that could be labelled as a candidate SLAF to proceed with the next step. SNPs with low minor allele frequencies $(<5 \%)$ and low call frequencies $(<85 \%)$ were deleted [32]. Finally, 33 samples and 2,309,777 SNPs remained for genome-wide association analysis (Additional file 7: Table S2).

\section{Phylogenetic analysis}

Based on the SNP genotype data from the 33 orchardgrass samples, a dendrogram was constructed in MEGA5 [41] using the neighbor-joining algorithm [42]. The structure of the orchardgrass population was analyzed using ADMIXTURE software based on SNP genotype data [43]. The pre-defined $\mathrm{K}$, which indicates the number of groups in a population, varied from 1 to 10 in ADMIXTURE models. A $\mathrm{K}$ value was selected when the estimate of $\ln \operatorname{Pr}(\mathrm{X} \mid \mathrm{K})$ peaked in the range of 1 to 10 subpopulations. Delta $K(\Delta \mathrm{K})$, as an ad hoc quantity related to the second order change in the log probability of the data with respect to the number of clusters, was considered as the most probable value of $\mathrm{K}$ according to the model choice criteria [42]. A PCA approach with the cluster software [44] was used to cluster the orchardgrass population.

\section{Evaluation of linkage disequilibrium and GWAS analysis}

The squared correlations $\left(\mathrm{r}^{2}\right)$ between all combinations of SNPs $(2,309,777)$ were used to evaluate the significance of pairwise linkage disequilibrium (LD) using Haploview [45] (http://www.softpedia.com/get/Science-CAD/Haploview .shtml). Each pair of SNPs was considered to have strong linkage if $r^{2}>0.8$. Based on SNP genotype data, the GWAS analysis was conducted using a GLM in the TASSEL software [43] as follows:

$$
\mathrm{Y}=\mathrm{X} \alpha+\mathrm{Q} \beta+\mathrm{K} \mu+\mathrm{eY}
$$

where $y$ is the phenotype value, $\mathrm{Q}$ is the population structure matrix calculated by the ADMIXTURE program, $X$ is the genotype matrix, $\alpha$ is the effect of genotype, $\beta$ is the effect of population structure, $\mu$ is the effect of kinship, and e is the residual error. Finally, each SNP locus was assigned a value associated with related traits. 


\section{Bulk analysis}

Two groups (HS and HR) were used to conduct a bulk analysis, and the procedures were followed as previously described [46]. To identify potential trait-retaled SNPs, we aligned the short reads obtained from the two DNA bulks to the reference genome (sequences from the HR group were regarded as reference genome) using bwa software [47], and alignment files were converted to SAM/BAM files using SAMtools [48]. SNP-index was calculated for all the SNP positions. We further excluded SNP positions with SNP-index of $<0.3$ from the two sequences to avoid spurious SNPs called due to alignment errors.

\section{Bioinformatics analysis of candidate regions}

The genetic data relating to rust traits of a $500-\mathrm{Kb}$ window surrounding each SNP were downloaded from Ensembl (http://ensemblgenomes.org/) and NCBI (http: //www.ncbi.nlm.nih.gov/). The NT databases were used to conduct pathway analysis and functional annotation for predicted genes [49].

\section{Conclusions}

In this study, SLAF-seq technology was used to conduct the GWAS in orchardgrass, and a number of 2,334,889 SLAF tags were produced to generate 2,309,777 SNPs. The GWAS showed that a total number of 4,604 SNPs were significantly $(P<0.01)$ associated with the rust trait, while a total number of 5,211 SNPs were filtered by bulk analysis. Based on bioinformatics analysis for GWAS results, four candidate genes, including cytochrome P450, Pc68LrkC4, prolamin, and $\mathrm{TaCab} 1$ were predicted involving in disease resistance, and the bulk analysis further confirmed cytochrome P450 and prolamin genes were reliable. Besides, further research should be done to determine how these two genes work for resisting diseases. The results can also provide basic information for MAS of rust-resistant lineages.

\section{Additional files}

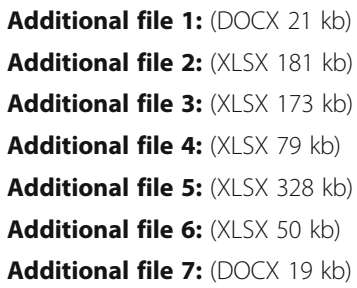

\section{Abbreviations}

ACD6: Cell death 6; G1: Group 1; G2: Group 2; GBS: Genotyping-bysequencing; GLM: General linear model; GWAS: Genome-wide association study; HR: High level of resistance of disease; HS: High susceptibility of disease; LD: Linkage disequilibrium; MAS: Marker-assisted selection; NT: Nucleotide sequences; PC: Principal component analysis; PC1: Principal Component 1; QTLs: Quantitative trait loci; RADseq: Restriction site-associated sequencing; RIL: Recombinant inbred line; SLAF-seq: Specific-locus amplified fragment sequencing; TaCab1: EF-hand $\mathrm{Ca}^{2+}$-binding protein

\section{Acknowledgments}

This work was supported by the National Basic Research Program of China (973 Program) (2014CB138705) and the National Natural Science Foundation of China, NSFC (31101760).

\section{Funding}

This work was supported by the National Basic Research Program of China (973 Program) (2014CB138705), Science and technology commission of livelihoods and social security project, and construction and demonstration promotion of grass and sheep matching model under whole barn feeding conditions for goats in Chongqing (cstc2016shmszx0473), Agriculture committee project and forage grass laboratory construction project of goat industrial technology system in Chongqing, and Funding project of innovation team construction in chongqing universities: modern production technology of beef (CXTDG201602003). And these four programs contribute to the design of the study, collection, analysis, interpretation of data, and in writing the manuscript.

\section{Availability of data and materials}

We have provided detailed information about materials and method in our manuscript, so we will not provide data and supporting materials in this section.

\section{Authors' contributions}

BZ and LH conceived and designed the experiments; HY and BZ performed the experiments; $H Y, X L$ and $B Z$ analyzed the data; $W Z, A Z, S Z$, JL and $H Y$ contributed reagents/materials/analysis tools; HY, WZ, AZ and SZ wrote the paper. All authors read and approved the final manuscript.

\section{Competing interests}

The authors declare that they have no competing interests.

\section{Consent for publication}

Not applicable.

Ethics approval and consent to participate

Not applicable.

\section{Author details \\ 'Department of Animal Science, Southwest University, Rongchang, Chongqing 402460, China. ${ }^{2}$ Department of Grassland Science, Animal Science and Technology College, Sichuan Agricultural University, Chengdu 611130, China. ${ }^{3}$ Agricultural College, Sichuan Agricultural University, Chengdu 611130, China. ${ }^{4}$ College of Life Science, China West Normal University, Nanchong 637009, China.}

Received: 20 August 2016 Accepted: 24 January 2017

Published online: 23 February 2017

\section{References}

1. Stewart AV, Ellison NW. The genus Dactylis; wealth of wild species: role in plant genome elucidation and improvement. New York: Springer; 2010.

2. Xie WG, Zhang XQ, Cai HW, Liu W, Peng Y. Genetic diversity analysis and transferability of cereal EST-SSR markers to orchardgrass (Dactylis glomerata L.). Biochem Syst Ecol. 2010;38(4):740-9.

3. Zeng B, Zhang Y, Huang LK, Jiang XM, Luo D, Yin GH. Genetic diversity of orchardgrass (Dactylis glomerata L.) germplasms with resistance to rust diseases revealed by Start Codon Targeted (SCOT) markers. Biochem Syst Ecol. 2014;54:96-102.

4. Tajimi A. Differentiation and maintenance of three graminicolous formae speciales of stem rust with clonal lines of orchardgrass, Dactylis glomerata $\mathrm{L}$. J Jpan Grassl Sci. 1975;20:26-30.

5. Miller TL, Carlson IT. Breeding for rust resistance in orchardgrass by phenotypic and phenotypic-genotypic selection. Crop Sci. 1982;22(6):1218-21.

6. Yan HD, Zeng B, Zhang XQ, Cheng L, Miller S, Huang LK. Screening orchardgrass (Dactylis glomerata L.) germplasm for high rust resistance and high summer survival rate in a subtropical climate. Grassl Sci. 2013;59(4):205-10.

7. Todesco M, Balasubramanian S, Hu TT, Traw MB, Horton M, Epple P, et al. Natural allelic variation underlying a major fitness trade-off in Arabidopsis thaliana. Nature. 2010;465(7298):632-6. 
8. Li Y, Huang Y, Bergelson J, Nordborg M, Borevitz JO. Association mapping of local climate-sensitive quantitative trait loci in Arabidopsis thaliana. PNAS. 2010;107(49):21199-204.

9. Tian F, Bradbury PJ, Brown PJ, Hung H, Sun Q, Flint-Garcia S, et al. Genomewide association study of leaf architecture in the maize nested association mapping population. Nat Genet. 2011;43(2):159-62.

10. Kollers S, Rodemann B, Ling J, Korzun V, Ebmeyer E, Argillier O, et al. Whole genome association mapping of Fusarium head blight resistance in European winter wheat (Triticum aestivum L.). PLoS One. 2013;8(2), e57500.

11. Begum H, Spindel JE, Lalusin A, Borromeo T, Gregorio G, Hernandez J, et al. Genome-wide association mapping for yield and other agronomic traits in an elite breeding population of tropical rice (Oryza sativa). PLoS One. 2015 10(3), e0119873.

12. Kumar V, Singh A, Mithra SVA, Krishnamurthy SL, Parida SK, Jain S, et al. Genome-wide association mapping of salinity tolerance in rice (Oryza sativa). DNA Res. 2015;22(2):133-45.

13. Yang WN, Guo ZL, Huang CL, Duan LF, Chen GX, Jiang N, et al. Combining high-throughput phenotyping and genome-wide association studies to reveal natural genetic variation in rice. Nat Commun. 2014;5.

14. Stracke S, Haseneyer G, Veyrieras J-B, Geiger HH, Sauer S, Graner A, et al. Association mapping reveals gene action and interactions in the determination of flowering time in barley. Theor Appl Genet. 2009;118(2):259-73.

15. Singh A, Reimer S, Pozniak CJ, Clarke FR, Clarke JM, Knox RE, et al. Allelic variation at Psy1-A1 and association with yellow pigment in durum wheat grain. Theor Appl Genet. 2009;118(8):1539-48.

16. Li YH, Zhang C, Gao ZS, Smulders MJM, Ma Z, Liu ZX, et al. Development of SNP markers and haplotype analysis of the candidate gene for rhg1, which confers resistance to soybean cyst nematode in soybean. Mol Breed. 2009;24(1):63-76.

17. Pasam RK, Sharma R, Malosetti M, van Eeuwijk FA, Haseneyer G, Kilian B, et al. Genome-wide association studies for agronomical traits in a world wide spring barley collection. BMC Plant Biol. 2012;12(1):16

18. Yu LX, Morgounov A, Wanyera R, Keser M, Singh SK, Sorrells M. Identification of Ug99 stem rust resistance loci in winter wheat germplasm using genome-wide association analysis. Theor Appl Genet. 2012;125(4):749-58.

19. Montilla-Bascón G, Rispail N, Sánchez-Martín J, Rubiales D, Mur LAJ, Langdon T, et al. Genome-wide association study for crown rust (Puccinia coronata f. sp. avenae) and powdery mildew (Blumeria graminis f. sp. avenae) resistance in an oat (Avena sativa) collection of commercial varieties and landraces. Front Plant Sci. 2015;6:1-11.

20. Qi ZM, Huang L, Zhu RS, Xin DW, Liu CY, Han X, et al. A high-density genetic map for soybean based on specific length amplified fragment sequencing. PLoS One. 2014;9(11), e114349.

21. Sun XW, Liu DY, Zhang XF, Li WB, Liu H, Hong WG, et al. SLAF-seq: an efficient method of large-scale de novo SNP discovery and genotyping using high-throughput sequencing. PLoS One. 2013;8(3), e58700.

22. Brachi B, Morris GP, Borevitz JO. Genome-wide association studies in plants: the missing heritability is in the field. Genome Biol. 2011;12(10):232.

23. Wang WH, Zhang T, Zhang GX, Wang JY, Han KP, Wang YJ, et al. Genomewide association study of antibody level response to NDV and IBV in Jinghai yellow chicken based on SLAF-seq technology. J Appl Genet. 2015;1-9.

24. Li B, Tian L, Zhang JY, Huang L, Han FX, Yan SR, et al. Construction of a high-density genetic map based on large-scale markers developed by specific length amplified fragment sequencing (SLAF-seq) and its application to QTL analysis for isoflavone content in Glycine max. BMC Genomics. 2014:15(1):1086.

25. Wei QZ, Wang YZ, Qin XD, Zhang YX, Zhang ZT, Wang J, et al. An SNPbased saturated genetic map and QTL analysis of fruit-related traits in cucumber using specific-length amplified fragment (SLAF) sequencing. BMC Genomics. 2014;15(1):1158.

26. Zhang $Y X$, Wang $L H$, Xin HG, Li DH, Ma CX, Ding $X$, et al. Construction of a high-density genetic map for sesame based on large scale marker development by specific length amplified fragment (SLAF) sequencing. BMC Plant Biol. 2013;13(1):141.

27. Xu XW, Xu RX, Zhu BY, Yu T, Qu WQ, Lu L, et al. A high-density genetic map of cucumber derived from Specific Length Amplified Fragment sequencing (SLAF-seq). Front Plant Sci. 2014;5.

28. Visscher PM, Brown MA, McCarthy MI, Yang J. Five years of GWAS discovery. Am J Hum Genet. 2012;90(1):7-24.

29. Zhao X, Han YP, Li YH, Liu DY, Sun MM, Zhao Y, et al. Loci and candidate gene identification for resistance to Sclerotinia sclerotiorum in soybean (Glycine max L. Merr.) viaassociation and linkage maps. Plant J. 2015;82(2):245-55.
30. Han Y, Zhao X, Liu D, Li Y, Lightfoot DA, Yang Z, et al. Domestication footprints anchor genomic regions of agronomic importance in soybeans. New Phytol. 2016;209(2):871-84.

31. Han YP, Zhao X, Cao GL, Wang Y, Li YH, Liu DY, et al. Genetic characteristics of soybean resistance to $\mathrm{HG}$ type 0 and $\mathrm{HG}$ type 1.2.3.5.7 of the cyst nematode analyzed by genome-wide association mapping. BMC Genom. 2015;16(1):1-11.

32. Purcell $S$, Neale B, Todd-Brown $K$, Thomas L, Ferreira MAR, Bender D, et al. PLINK: a tool set for whole-genome association and population-based linkage analyses. Am J Hum Genet. 2007;81(3):559-75.

33. Pritchard JK, Stephens M, Rosenberg NA, Donnelly P. Association mapping in structured populations. Am J Hum Genet. 2000;67(1):170-81.

34. Hamblin MT, Close TJ, Bhat PR, Chao S, Kling JG, Abraham KJ, et al. Population structure and linkage disequilibrium in US barley germplasm: implications for association mapping. Crop Sci. 2010;50(2):556-66.

35. Urlacher VB, Lutz-Wahl S, Schmid RD. Microbial P450 enzymes in biotechnology. Appl Microbiol Biotechnol. 2004;64(3):317-25.

36. Rinaldi C, Kohler A, Frey P, Duchaussoy F, Ningre N, Couloux A, et al. Transcript profiling of poplar leaves upon infection with compatible and incompatible strains of the foliar rust Melampsora larici-populina. Plant Physiol. 2007;144(1):347-66.

37. Feuillet C, Schachermayr G, Keller B. Molecular cloning of a new receptorlike kinase gene encoded at the LrlO disease resistance locus of wheat. Plant J. 1997;11(1):45-52.

38. Sun G, Fahima T, Korol A, Turpeinen T, Grama A, Ronin Y, et al. Identification of molecular markers linked to the Yr15 stripe rust resistance gene of wheat originated in wild emmer wheat, Triticum dicoccoides. Theor Appl Genet. 1997;95(4):622-8.

39. Peng JH, Fahima T, Röder MS, Li YC, Dahan A, Grama A, et al. Microsatellite tagging of the stripe-rust resistance gene $\mathrm{YrH} 52$ derived from wild emmer wheat, Triticum dicoccoides, and suggestive negative crossover interference on chromosome 1B. Theor Appl Genet. 1999;98(6-7):862-72.

40. Feng $H$, Wang XM, Sun YF, Wang XJ, Chen XM, Guo J, et al. Cloning and characterization of a calcium binding EF-hand protein gene TaCab1 from wheat and its expression in response to Puccinia striiformis f. sp. tritici and abiotic stresses. Mol Biol Rep. 2011;38(6):3857-66.

41. Saitou N, Nei M. The neighbor-joining method: a new method for reconstructing phylogenetic trees. Mol Biol Evolution. 1987;4(4):406-25.

42. Eickmeyer $K$, Huggins $P$, Pachter L, Yoshida R. On the optimality of the neighbor-joining algorithm. Algorithms Mol Biol. 2008;3(1):1.

43. de Hoon MJL, Imoto S, Nolan J, Miyano S. Open source clustering software. Bioinform. 2004;20(9):1453-4.

44. Groth D, Hartmann S, Klie S, Selbig J. Principal components analysis. Computational Toxicology: Volume II. 2013;2013:527-47.

45. Barrett JC, Fry B, Maller JDMJ, Daly MJ. Haploview: analysis and visualization of LD and haplotype maps. Bioinform. 2005;21(2):263-5.

46. Takagi H, Abe A, Yoshida K, Kosugi S, Natsume S, Mitsuoka C, et al. QTL-seq: rapid mapping of quantitative trait loci in rice by whole genome resequencing of DNA from two bulked populations. Plant J. 2013;74(1):174-83.

47. Li H, Durbin R. Fast and accurate long-read alignment with BurrowsWheeler transform. Bioinformatics. 2010;25(5):1754-60.

48. Li H. The Sequence Alignment-Map format and SAMtools. Bioinformatics. 2009;25(16):2078-9.

49. Pruitt KD, Tatusova T, Maglott DR. NCBI Reference Sequence (RefSeq): a curated non-redundant sequence database of genomes, transcripts and proteins. Nucleic Acid Res. 2005;33 suppl 1:D501-4. 